\title{
Three-dimensional pyramid microlasers
}

\author{
Stefan Bittner ${ }^{1,2}$, Melissa A. Guidry ${ }^{1}$, Yalei Song ${ }^{3}$, Clément Lafargue ${ }^{1}$, Roch Sobcyk ${ }^{1}$, Dominique \\ Decanini $^{4}$, Barbara Dietz ${ }^{3}$, Liang Huang ${ }^{3}$, Joseph Zyss ${ }^{1}$, Alain Grigis ${ }^{5}$, Mélanie Lebental ${ }^{1}$ \\ 1. Laboratoire de Photonique Quantique et Moléculaire, CNRS UMR 8537 ENS Paris-Saclay, CentraleSupélec, F-94235 Cachan, France \\ 2. Chaire Photonique, LMOPS, CentraleSupélec, University Paris-Saclay, F-57070 Metz, France \\ 3. School of Physical Science and Technology, and Key Laboratory for Magnetism and Magnetic Materials of MOE, \\ Lanzhou University, Lanzhou, Gansu 730000, China \\ 4. Centre de Nanosciences et de la Nanotechnologie, CNRS, University Paris-Sud, University Paris-Saclay, F-91460 Marcoussis, France \\ 5. Laboratoire d'Analyse, Géometrie et Applications, CNRS UMR 7539, University Sorbonne Paris City, University Paris 13, \\ Institut Galilée, F-93430 Villetaneuse, France
}

Direct laser writing based on two-photon absorption allows the fabrication of almost arbitrary threedimensional (3D) structures with sub-micron precision. This enables innovative designs for optical microcavities and -lasers in view of applications such as chemical sensing. While the complexity of 3D structures enables new functionalities, it also increases the difficulty of understanding and designing resonator properties. Semiclassical methods based on periodic orbits (POs) are a promising solution. Experiments with flat 2D organic microlasers have shown the importance and usefulness of POs to understand and control their lasing properties [1], however, the extension of these methods to three dimensions remains a challenge.

We present measurements of the lasing spectra as well as emission intensity distributions of 3D pyramidshaped microlasers [2]. High-quality pyramid microlasers with a side length and height of $50 \mu \mathrm{m}$ were fabricated from a photo resist doped with a laser dye [see Fig. 1(a)]. Individual cavities are pumped optically with 0.5 -ns pulses from a frequency-doubled Nd:YAG laser at $532 \mathrm{~nm}$. Figure 1(b) shows the lasing spectra with vertical and horizontal polarization (with respect to the pyramid base) which both consist of a comb of equidistant lasing peaks. The free spectral range of the spectra is inversely proportional to the length of the underlying PO, which was identified as the so-called folded diamond orbit shown in Fig. 1(c). This orbit is reflected from the pyramid base four times as well as once from each lateral facet and resembles a diamond when viewed from the top. The measured emission intensity distributions are explained by the geometry of this PO as well.

Previous studies of 3D microlasers only found modes based on 2D orbits [3], i.e., POs that are confined to a single plane. The folded diamond PO, in contrast, is a non-planar and hence genuinely three-dimensional PO. Its $3 \mathrm{D}$ geometry has important consequences for the stability of the orbit and influences the polarization properties of the lasing modes. Since rotations do not commute in 3D, the initial polarization of a wave following the PO is rotated by the so-called screw angle, which is a geometric property of the PO. The different reflection phases for $s$ - and $p$-polarized waves at the dielectric interfaces furthermore change the initial polarization. Therefore, the geometry of the cavity and in extension the lasing PO allows to control the polarization of the lasing modes.

The pyramid cavities lasing on a 3D periodic orbit are a first example of the potential of 3D microcavities and -lasers. The 3D geometry allows new functionalities such as controlling the polarization state of modes for applications requiring special polarization states in, e.g., chemical sensing or spintronics.
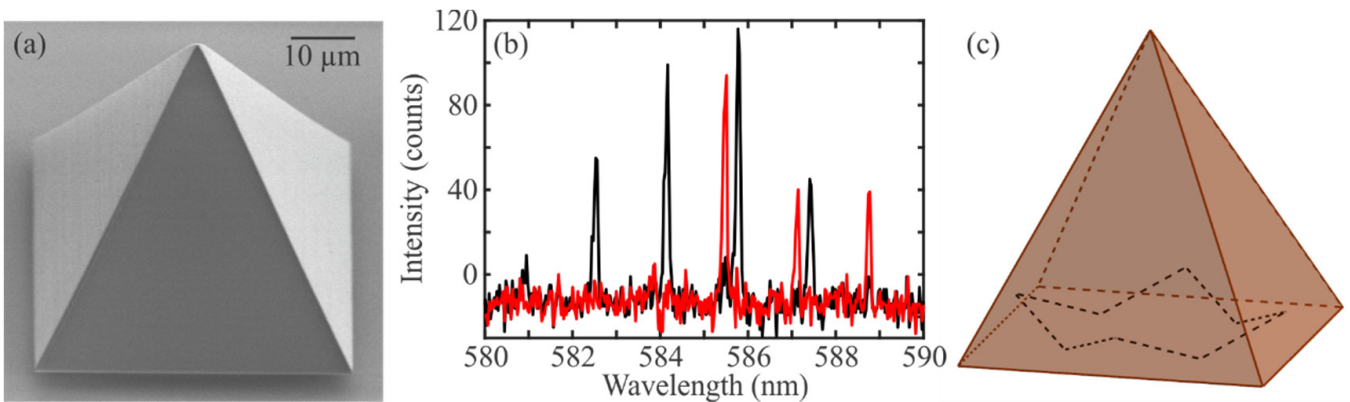

Fig. 1 Organic pyramid-shaped microlaser. (a) SEM image of a pyramid with $50 \mu \mathrm{m}$ side length and height. (b) Equidistant lasing spectra of horizontally (black) and vertically (red) polarized modes. (c) Folded diamond periodic orbit (black dashed line) in a pyramid with square base.

\section{Example References}

[1] E. Bogomolny et al., "Trace formula for dielectric cavities II: Regular, pseudo-integrable, and chaotic examples", Phys. Rev. E 83, $036208(2011)$

[2] M. A. Guidry et al., "Three-dimensional micro-billiard lasers: the square pyramid", arXiv:1901.05381 (2019).

[3] V. Chen et al., "Three-dimensional organic microlasers with low lasing thresholds fabricated by multiphoton and UV lithography", Opt. Express 22, 12316 (2014). 